\title{
NEUMANN EIGENVALUE ESTIMATE ON A COMPACT RIEMANNIAN MANIFOLD
}

\author{
ROGER CHEN
}

(Communicated by Jonathan M. Rosenberg)

\begin{abstract}
In their article, P. Li and S. T. Yau give a lower bound of the first Neumann eigenvalue in terms of geometrical invariants for a compact Riemannian manifold with convex boundary. The purpose of this paper is to generalize their result to a compact Riemannian manifold with possibly nonconvex boundary.
\end{abstract}

\section{INTRODUCTION}

Let $M^{n}$ be an $n$-dimensional compact Riemannian manifold with boundary $\partial M$. In local coordinates $\left(x^{1}, x^{2}, \ldots, x^{n}\right)$, the Riemannian metric is given by

$$
d s^{2}=\sum_{i, j=1}^{n} g_{i j} d x^{i} d x^{j}
$$

One defines on $M$ a second order elliptic differential operator by

$$
\Delta=\frac{1}{\sqrt{g}} \sum_{i, j=1}^{n} \frac{\partial}{\partial x^{i}}\left(\sqrt{g g}^{i j} \frac{\partial}{\partial x^{j}}\right),
$$

where $\left(g^{i j}\right)=\left(g_{i j}\right)^{-1}$ and $g=\operatorname{det}\left(g_{i j}\right)$, which is known as the Laplace operator. The purpose of this paper is the study of eigenvalues of the Laplace operator. More specifically, we study the following problem:

Assume that $\partial M \neq \varnothing$, we adopt an "interior rolling $\varepsilon$-ball" condition on $\partial M$ to consider the following Neumann eigenvalue problem on $M^{n}$ :

$$
\left\{\begin{array}{l}
\Delta h=-\eta h \\
\frac{\partial h}{\partial \nu} \equiv 0 \text { on } \partial M
\end{array}\right.
$$

where $\nu$ is the unit outward normal vector to the boundary $\partial M$.

Definition 1.1. Let $\partial M$ be the boundary of a compact Riemannian manifold $M^{n}$. Then $\partial M$ satisfies the "interior rolling $\varepsilon$-ball" condition if for each point

Received by the editors December 8, 1988 and, in revised form, April 17, 1989.

1980 Mathematics Subject Classification (1985 Revision). Primary 53-XX, 58G20, 58G25. 
$p \in \partial M$ there is a geodesic ball $B_{q}(\varepsilon / 2)$, centered at $q \in M$ with radius $\varepsilon / 2$, such that

$$
p=B_{q}(\varepsilon / 2) \cap \partial M \text { and } B_{q}(\varepsilon / 2) \subset M .
$$

It is well known that the set of eigenvalues $\left\{\eta_{k}\right\}$ of (1.3) are nonnegative and can be arranged in a nondecreasing order of magnitude as follows:

$$
0=\eta_{0}<\eta_{1} \leq \eta_{3} \leq \cdots \leq \eta_{m} \leq \cdots .
$$

By the compactness of $M^{n}$, it is known that those functions which satisfy (1.3) with eigenvalue $\eta_{0}$ are constants. The first nonzero eigenvalue $\eta_{1}$ in the problem (1.3) is hence characterized as the optimal constant in the Poincaré inequality:

$$
\eta_{1} \int f^{2} \leq \int|\nabla f|^{2}
$$

for all $f \in H_{1}^{2}(M)$ such that $\int_{M} f=0$. Due to the importance of Poincare inequality for analysis on manifolds, one wishes to obtain optimal quantitative estimates for the first eigenvalue $\eta_{1}$ from below in terms of geometric elements. Classically, for domains in $\mathbf{R}^{n}$, lower estimates for $\eta_{1}$ were established by Payne-Weinberger [4] and Payne-Stakgold [3], etc. For general compact manifolds with convex boundary, the lower estimates of $\eta_{1}$ were obtained by Li-Yau [2]. Using a method similar to that of Li-Yau [2], we have the following:

Theorem 1.1. Let $M^{n}$ be a compact Riemannian manifold with boundary $\partial M$. Let $\partial M$ satisfy the "interior rolling $\varepsilon$-ball" condition. Let $K$ and $H$ be nonnegative constants such that the Ricci curvature Ric of $_{M}$ is bounded below by $-K$ and the second fundamental form elements of $\partial M$ is bounded below by $-H$. By choosing $\varepsilon$ "small", we have

$$
\frac{1}{(1+H)^{2}}\left[\frac{1-\alpha^{2}}{4(n-1) d^{2}} B^{2}-C\right] \exp (-B) \leq \eta_{1}
$$

where $\alpha$ and $\varepsilon$ are positive constants less than 1 .

$$
\begin{aligned}
& d=\text { diameter of } M^{n}, \\
& B=1+\left[1+\frac{\left.4(n-1) d^{2} C\right)}{1-\alpha^{2}}\right]^{1 / 2}, \\
& C=(1+H) C_{1}+\frac{\left[(2 n-3)^{2}+(4 n-5) \alpha^{2}\right] H^{2}}{(n-1) \varepsilon^{2} \alpha^{2}}+(H+1)^{2} K,
\end{aligned}
$$

and

$$
C_{1}=\frac{2(n-1) H(3 H+1)(H+1)}{\varepsilon}+\frac{H+H^{2}}{\varepsilon^{2}} .
$$

Remark 1 . When the boundary $\partial M$ is convex, our estimate implies the estimate, obtained by Li-Yau [2, Theorem 9]. 
Remark 2. In our estimate, the choice of $\varepsilon$ depends on the upper bound of the sectional curvature near the boundary. We do not know whether we can determine the upper bound of $\varepsilon$ without using the curvature bound near the boundary. The upper bound of $\varepsilon$ is given by (2.16) and (2.17).

In $\S 2$, we shall give a gradient estimate which is essential in a proof of the main result. In $\S 4$, we shall give a proof of Theorem 1.1. In $\S 3$, a counterexample is given which will demonstrate that the "interior rolling $\varepsilon$-ball" condition is definitely necessary for $\eta_{1}$ being bounded away from 0 .

\section{A GRADIENT ESTIMATE}

Throughout this section, $M^{n}$ is assumed to be an $n$-dimensional compact Riemannian manifold with boundary $\partial M$. Let $K, H$ be nonnegative constants which were defined in $\S 1$. Let $f$ be a function which satisfies (1.3) with $\eta=\eta_{1}$; i.e., $f$ is an eigenfunction of (1.3) with eigenvalue $\eta_{1}$.

In this section, our goal is the study of the solution of equation (1.3) using maximal principle.

Let us first recall some general facts concerning a Riemannian manifold. Let $\left\{e_{i}\right\}$ be a local frame field of a Riemannian manifold $M^{n}$ and $\left\{\omega_{i}\right\}$ be the corresponding dual frame field. Then the structure equations of $M^{n}$ are given by

$$
\begin{aligned}
d \omega_{j} & =\sum_{i=1}^{n} \omega_{i j} \wedge \omega_{j}, \quad \omega_{i j}=-\omega_{j i}, \\
d \omega_{i j} & =\sum_{k=1}^{n} \omega_{i k} \wedge \omega_{k j}+\frac{1}{2} \sum_{k, l}^{n} R_{i j k l} \omega_{l} \wedge \omega_{k} .
\end{aligned}
$$

For any $C^{2}$-function $p(x)$ defined on $M^{n}$, we may define its gradient and Hessian by the following formulas:

$$
\begin{aligned}
d p & =\sum_{i=1}^{n} p_{i} \omega_{i}, \\
\sum_{j=1}^{n} p_{i j} \omega_{j} & =d p_{i}+\sum_{j=1}^{n} p_{j} \omega_{j i}
\end{aligned}
$$

and the covariant derivatives of $p_{i j}$ are defined by

$$
\sum_{k=1}^{n} p_{i j k} \omega_{k}=d p_{i j}+\sum_{k=1}^{n} p_{k j} \omega_{k i}+\sum_{k=1}^{n} p_{i k} \omega_{k j}
$$

By exteriorly differentiating (2.4), we get the following commutational formula:

$$
p_{i j k}-p_{i k j}=\sum_{l=1}^{n} p_{l} R_{l i j k}
$$


We have the following:

Theorem 2.1. Let $M^{n}$ be an n-dimensional compact Riemannian manifold with boundary $\partial M$ satisfying the "interior rolling $\varepsilon$-ball" condition. Let $f$ be a solution of equation (1.4) with $\eta=\eta_{1}$. If $\mu>1$ is any constant, and $\varepsilon$ is "small", then

$$
\begin{array}{r}
\frac{|\nabla f|^{2}}{(\mu \sup f-f)^{2}} \leq \max \left\{\frac{4(n-1)}{1-\alpha^{2}}\left[C+(H+1)^{2} \eta_{1}\left\|\frac{\mu \sup f}{\mu \sup f-f}\right\|_{\infty}\right],\right. \\
\left.\frac{\sqrt{8}(H+1)^{2}}{\sqrt{1-\alpha^{2}}} \eta_{1}\left\|\frac{f}{\mu \sup f-f}\right\|_{\infty}\right\},
\end{array}
$$

where $\alpha$ and $\varepsilon$ are positive constants less than 1 , and

$$
\begin{aligned}
C & =(1+H) C_{1}+\frac{\left[(2 n-3)^{2}+(4 n-5) \alpha^{2}\right] H^{2}}{(n-1) \varepsilon^{2} \alpha^{2}}+(1+H)^{2} K, \\
C_{1} & =\frac{2(n-1) H(3 H+1)(1+H)}{\varepsilon}+\frac{H+H^{2}}{\varepsilon^{2}} .
\end{aligned}
$$

Remark 1 . When the boundary $\partial M$ is convex, our theorem implies the gradient estimate obtained in Li-Yau [2, Theorem 3].

Remark 2. In our estimate, $\varepsilon$ is chosen to be a positive constant less than 1 and is dependent on the upper bound of the sectional curvature of the manifold near the boundary. The upper bound of $\varepsilon$ is given by (2.16) and (2.17).

Proof. Let $\psi(r)$ be a nonnegative $C^{2}$ function defined on $[0, \infty)$ such that

$$
\psi(r) \begin{cases}\leq H & \text { if } r \in\left[0, \frac{1}{2}\right), \\ =H & \text { if } r \in[1, \infty),\end{cases}
$$

with

$$
\psi(0)=0, \quad 2 H \geq \psi^{\prime}(r) \geq 0, \quad \psi^{\prime}(0)=H
$$

and

$$
\psi^{\prime \prime}(r) \geq-H
$$

Let

$$
\phi(x)=\psi\left(\frac{r(x)}{\varepsilon}\right),
$$

where $r(x)$ denotes distance between boundary $\partial M$ and $x \in M$. For $\mu>1$, we define the function

$$
G(x)=(1+\phi)^{2} \frac{|\nabla f|^{2}}{(\mu \sup f-f)^{2}} .
$$

By the compactness of $M$, there is a point $p \in M$ such that $G$ achieves its supremum. We may assume that $G(p)>0$, or else the theorem follows trivially. Suppose that $p$ is a boundary point of $\partial M$. At $p$ be may choose an 
orthonormal frame $e_{1}, \cdots, \varepsilon_{n}$ such that $\varepsilon_{n}=\frac{\partial}{\partial \nu}$, where $\nu$ is the unit outward normal vector to $\partial \mathbf{M}$. Then we have

$$
0 \leq \frac{\partial G}{\partial \nu}(p)
$$

This gives

$$
\begin{aligned}
0 & \leq \frac{\partial \phi / \partial \nu}{1+\phi}+\frac{\sum_{i=1}^{n} f_{i} f_{i \nu}}{|\nabla f|^{2}}-\frac{f \nu}{\mu \sup f-f} \\
& =-\frac{H}{\varepsilon}+\frac{\sum_{i=1}^{n-1} f_{i} f_{i \nu}}{|\nabla f|^{2}} .
\end{aligned}
$$

If $h_{i j}$ are the second fundamental form elements of $\partial M$, then by a direct computation one shows that

$$
f_{i \nu}=-\sum_{j=1}^{n-1} h_{i j} f_{j} \quad \text { for } 1 \leq i \leq n-1,
$$

where we used the fact that $f_{\nu} \equiv 0$ on $\partial M$. Together with (2.9), we have

$$
\begin{aligned}
0 & \leq-\frac{H}{\varepsilon}-\frac{\sum_{i, j=1}^{n-1} h_{i j} f_{i} f_{j}}{|\nabla f|^{2}} \\
& \leq-\frac{H}{\varepsilon}+H \\
& <0,
\end{aligned}
$$

which is a contradiction, as we choose $\varepsilon$ to be smaller than 1 . Hence $G(x)$ cannot attain its maximum at the boundary point. Therefore $p$ has to be an interior point of $M$. Hence at $p$

$$
\nabla G=0
$$

and

$$
\Delta G \leq 0
$$

This gives

$$
0=\frac{\psi^{\prime} r_{j}}{\varepsilon(1+\phi)}+\frac{\sum_{i=1}^{n} f_{i} f_{i j}}{|\nabla f|^{2}}+\frac{f_{j}}{\mu \sup f-f},
$$

and

$$
\begin{aligned}
0 \geq & \frac{\Delta \phi}{1+\phi}-\frac{\left(\psi^{\prime}\right)^{2}}{\varepsilon^{2}(1+\phi)^{2}}+\left(\sum_{i, j=1}^{n} f_{i j}^{2}+\sum_{i, j=1}^{n} f_{i} f_{i j j}\right) /|\nabla f|^{2} \\
& -\frac{2 \sum_{j=1}^{n}\left(\sum_{i=1}^{n} f_{i} f_{i j}\right)^{2}}{|\nabla f|^{4}}+\frac{\Delta f}{\mu \sup f-f}+\frac{|\nabla f|^{2}}{(\mu \sup f-f)^{2}} .
\end{aligned}
$$


To compute $\Delta \phi$, we let $\partial M(\varepsilon)=\{x \in M \mid r(x) \leq \varepsilon\}$ and $K_{\varepsilon}$ be the upper bound of the sectional curvature in $\partial M(\varepsilon)$. We may choose $\varepsilon$ to be small so that

$$
\sqrt{K_{\varepsilon}} \tan \left(\varepsilon \sqrt{K_{\varepsilon}}\right) \leq \frac{H}{2}+\frac{1}{2}
$$

and

$$
\frac{H}{\sqrt{K_{\varepsilon}}} \tan \left(\varepsilon \sqrt{K_{\varepsilon}}\right) \leq \frac{1}{2} .
$$

By using an index comparison theorem in Riemannian geometry [5, p. 347], one can show that if $x \in \partial M(\varepsilon)$, we have

$$
\begin{aligned}
\Delta r & \geq-(n-1) \frac{\varepsilon H+\varepsilon \sqrt{K_{\varepsilon}} \tan \left(\varepsilon \sqrt{K_{\varepsilon}}\right)}{\varepsilon-\varepsilon \frac{H}{\sqrt{K_{\varepsilon}}} \tan \left(\varepsilon \sqrt{K_{\varepsilon}}\right)} \\
& \geq-(n-1)(3 H+1) .
\end{aligned}
$$

Then we have

$$
\begin{aligned}
\Delta \phi & =\frac{1}{\varepsilon} \psi^{\prime} \Delta r+\frac{1}{\varepsilon^{2}} \psi^{\prime \prime}|\nabla r|^{2} \\
& \geq-\frac{2(n-1) H(3 H+1)}{\varepsilon}-\frac{H}{\varepsilon^{2}} \\
& =-C_{1} .
\end{aligned}
$$

At $p$, we may choose an orthonormal frame $\left\{e_{i}\right\}$ such that $f_{1}(p)=|\nabla f|(p)$. By using (2.6) and (2.14), we also have, at $p$,

$$
f_{j 1 j}-f_{j j 1}=\sum_{l=1}^{n} f_{l} R_{l j 1 j}
$$

and

$$
f_{1 j}=-\frac{\psi^{\prime} f_{1} r_{j}}{\varepsilon(1+\phi)}-\frac{f_{1} f_{j}}{\mu \sup f-f} .
$$

Substituting these and (2.18) into (2.15), we have

$$
\begin{aligned}
0 \geq & -\frac{C_{1}}{1+\phi}-\frac{\left[1+r_{1}^{2}\right]\left(\psi^{\prime}\right)^{2}}{\varepsilon^{2}(1+\phi)^{2}}-\frac{2 \psi^{\prime} f_{1} r_{1}}{\varepsilon(1+\phi)(\mu \sup f-f)} \\
& +\frac{\sum_{i>1} f_{i i}^{2}}{f_{1}^{2}}+R i c_{11}-\frac{\eta_{1} \mu \sup f}{\mu \sup f-f}, \\
\geq & -\frac{C_{1}}{1+\phi}-\frac{2\left(\psi^{\prime}\right)^{2}}{\varepsilon^{2}(1+\phi)^{2}}-\frac{2 \psi^{\prime} f_{1} r_{1}}{\varepsilon(1+\phi)(\mu \sup f-f)} \\
& +\frac{\sum_{i>1} f_{i i}^{2}}{f_{1}^{2}}+R i c_{11}-\frac{\eta_{1} \mu \sup f}{\mu \sup f-f} .
\end{aligned}
$$


It is also clear that

$$
\begin{aligned}
\sum_{i>1} f_{i i}^{2} & \geq \frac{1}{n-1}\left(\sum_{i>1} f_{i i}\right)^{2} \\
& \geq \frac{f_{11}^{2}}{2(n-1)}-\frac{(\Delta f)^{2}}{n-1}
\end{aligned}
$$

Then we have

$$
\begin{aligned}
\sum_{i>1} f_{i i}^{2} \geq & \frac{f_{1}^{4}}{2(n-1)(\mu \sup f-f)^{2}}+\frac{f_{1}^{3} \psi^{\prime} r_{1}}{\varepsilon(n-1)(1+\phi)(\mu \sup f-f)} \\
& +\frac{\left(\psi^{\prime}\right)^{2} f_{1}^{2} r_{1}^{2}}{2 \varepsilon^{2}(n-1)(1+\phi)^{2}}-\frac{\eta_{1}^{2} f^{2}}{n-1} .
\end{aligned}
$$

Substituting (2.21) into (2.19), we have

$$
\begin{aligned}
0 \geq & \frac{f_{1}^{2}}{2(n-1)(\mu \sup f-f)^{2}}-\frac{(2 n-3) f_{1} \psi^{\prime} r_{1}}{\varepsilon(n-1)(1+\phi)(\mu \sup f-f)}-\frac{C_{1}}{1+\phi} \\
& -\frac{4(n-1)\left(\psi^{\prime}\right)^{2}-\left(\psi^{\prime}\right)^{2} r_{1}^{2}}{2 \varepsilon^{2}(n-1)(1+\phi)^{2}}-K-\frac{\eta_{1} \mu \sup f}{\mu \sup f-f}-\frac{\eta_{1}^{2} f^{2}}{(n-1) f_{1}^{2}} .
\end{aligned}
$$

It is clear that

$$
\begin{gathered}
\frac{\alpha^{2} f_{1}^{2}}{2(n-1)(\mu \sup f-f)^{2}}-\frac{(2 n-3) f_{1} \psi^{\prime} r_{1}}{\varepsilon(n-1)(1+\phi)(\mu \sup f-f)} \\
\geq-\frac{(2 n-3)^{2}\left(\psi^{\prime}\right)^{2}\left(r_{1}\right)^{2}}{2 \varepsilon^{2} \alpha^{2}(n-1)(1+\phi)^{2}}, \\
\geq-\frac{(2 n-3)^{2}\left(\psi^{\prime}\right)^{2}}{2 \varepsilon^{2} \alpha^{2}(n-1)(1+\phi)^{2}} .
\end{gathered}
$$

Substituting this into (2.22), we have

$$
\begin{aligned}
0 \geq & \left(\frac{1-\alpha^{2}}{2(n-1)}\right) \frac{f_{1}^{2}}{(\mu \sup f-f)^{2}}+\frac{\left[\alpha^{2}-(2 n-3)^{2}\right]\left(\psi^{\prime}\right)^{2}\left(r_{1}\right)^{2}}{2 \varepsilon^{2} \alpha^{2}(n-1)(1+\phi)^{2}} \\
& -\frac{2\left(\psi^{\prime}\right)^{2}}{\varepsilon^{2}(1+\phi)^{2}}-\frac{C_{1}}{1+\phi}-K-\frac{\eta_{1} \mu \sup f}{\mu \sup f-f}-\frac{\eta_{1}^{2} f^{2}}{(n-1) f_{1}^{2}} \\
\geq & \left(\frac{1-\alpha^{2}}{2(n-1)}\right) \frac{f_{1}^{2}}{(\mu \sup f-f)^{2}}-\frac{\left[(2 n-3)^{2}-\alpha^{2}\right]\left(\psi^{\prime}\right)^{2}}{2 \varepsilon^{2} \alpha^{2}(n-1)(1+\phi)^{2}} \\
& -\frac{2\left(\psi^{\prime}\right)^{2}}{\varepsilon^{2}(1+\phi)^{2}}-\frac{C_{1}}{1+\phi}-K-\frac{\eta_{1} \mu \sup f}{\mu \sup f-f}-\frac{\eta_{1}^{2} f^{2}}{(n-1) f_{1}^{2}} .
\end{aligned}
$$


Hence

(2.24)

$$
\begin{gathered}
0 \geq \frac{\left(1-\alpha^{2}\right) f_{1}^{2}}{2(n-1)(\mu \sup f-f)^{2}}-\left[\frac{\left[(2 n-3)^{2}+(4 n-5) \alpha^{2}\right]\left(\psi^{\prime}\right)^{2}}{2 \varepsilon^{2} \alpha^{2}(n-1)(1+\phi)^{2}}+\frac{C_{1}}{1+\phi}\right. \\
\left.+K+\frac{\eta_{1} \mu \sup f}{\mu \sup f-f}\right]-\frac{\eta_{1}^{2} f^{2}}{(n-1) f_{1}^{2}} .
\end{gathered}
$$

Multiplying through by $(1+\phi)^{4} \frac{f_{1}^{2}}{(\mu \sup f-f)^{2}},(2.24)$ becomes

$$
\begin{array}{r}
0 \geq \frac{1-\alpha^{2}}{2(n-1)} G^{2}-\left[\frac{\left[(2 n-3)^{2}+(4 n-5) \alpha^{2}\right]\left(\psi^{\prime}\right)^{2}}{2 \varepsilon^{2} \alpha^{2}(n-1)}+(1+\phi) C_{1}+(1+\phi)^{2} K\right. \\
\left.+\frac{\eta_{1} \mu \sup f(1+\phi)^{2}}{\mu \sup f-f}\right] G-\frac{\eta_{1}^{2} f^{2}(1+\phi)^{4}}{(n-1)(\mu \sup f-f)^{2}} \\
\geq \frac{1-\alpha^{2}}{2(n-1)} G^{2}-\left[\frac{2\left[(2 n-3)^{2}+(4 n-5) \alpha^{2}\right] H^{2}}{\varepsilon^{2} \alpha^{2}(n-1)}+(1+H) C_{1}+(1+H)^{2} K\right. \\
\left.+\frac{(1+H)^{2} \eta_{1} \mu \sup f}{\mu \sup f-f}\right] G-\frac{(1+H)^{4} \eta_{1}^{2} f^{2}}{(n-1)(\mu \sup f-f)^{2}} .
\end{array}
$$

This implies Theorem 2.1.

\section{A COUNTEREXAMPLE}

In this section, we shall show that the "interior rolling $\varepsilon$-ball" condition is necessary for $\eta_{1}$ being bounded away from zero. We consider the following well-known example of E. Calabi [1]. For the sake of completeness, we will describe the example here.

Example 3.1. Let $\Omega \subset \mathbf{R}^{2}$ be a domain as in Figure 1. The rectangle connecting the two disks is to be thought of as having fixed length $l$ and variable width $w$.

Let $f$ be a function which is equal to $c$ on the right-hand disk, $-c$ on the left-hand disk and changes linearly from $c$ to $-c$ across the rectangle. $(c$ is chosen to that $\int_{\Omega} f^{2}=1$.) Then $\int_{\Omega} f=0$ and

It is clear that

$$
\|\nabla f\|= \begin{cases}0 & \text { on the disks } \\ \frac{2 c}{T} & \text { on the rectangle. }\end{cases}
$$

$$
\begin{aligned}
\eta_{1} & =\inf _{h \in H_{1}^{2}(\Omega) \text { such that }} \int_{\Omega} h=0 \frac{\int_{\Omega}|\nabla h|^{2}}{\int_{\Omega} h^{2}} \\
& \leq \int_{\Omega}|\nabla f|^{2} \\
& =\frac{2 c}{l} w l \rightarrow 0, \quad \text { as } w \rightarrow 0 .
\end{aligned}
$$




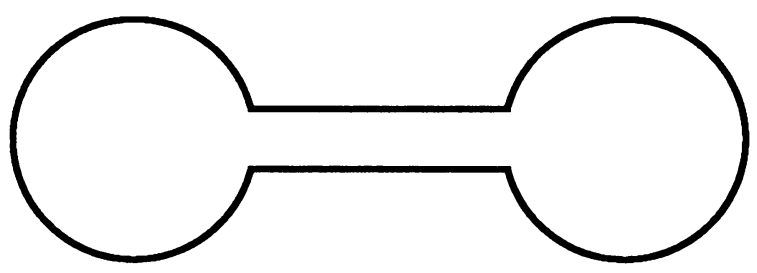

Figure 1. DuMbBell

This example shows that in bounding $\eta_{1}$ from below, it is necessary to consider the "interior rolling $\varepsilon$-ball" condition.

\section{Proof of Theorem 1.1}

In this section, we let $f$ be a function which satisfies (1.3) with $\eta=\eta_{1}$; i.e., $f$ is an eigenfunction of (1.3) with eigenvalue $\eta_{1}$. We denote $N$ the nodal set of $f$; i.e., $N=\{x \in \bar{M} \mid f(x)=0\}$.

Proof. From Theorem 2.1, we know that

$$
\begin{array}{r}
\frac{|\nabla f|}{\mu \sup f-f} \leq \max \left\{\frac{\sqrt{4(n-1)}}{\sqrt{1-\alpha^{2}}}\left[C+\frac{(H+1)^{2} \eta_{1} \mu}{\mu-1}\right]^{1 / 2},\right. \\
\left.\frac{\sqrt{S}(H+1)}{\sqrt{1-\alpha^{2}}} \frac{\eta_{1}^{1 / 2}}{(\mu-1)^{1 / 2}}\right\}
\end{array}
$$

for any constant $\mu>1$.

However, since $f$ satisfies

$$
\int_{M} f=0
$$

and

$$
f \not \equiv 0,
$$

this implies that the nodal set $N$ of $f$ divides $\bar{M}$ into two parts. If $x \in \bar{M}$ is a point where $f$ achieves its supremum and $\gamma$ is a shortest geodesic joining $x$ and $N$, then $\gamma$ has length at most diameter of $M$. Integrating (4.1) along $\gamma$, we have

$$
\begin{aligned}
\log \frac{\mu}{\mu-1} & \leq \int_{\gamma} \frac{|\nabla f|}{\mu \sup f-f} \\
& \leq \frac{4 \sqrt{(n-1)}}{1-\alpha^{2}}\left[C+\frac{(H+1)^{2} \mu \eta_{1}}{\mu-1}\right]^{\frac{1}{2}} d .
\end{aligned}
$$


Hence

$$
\frac{\mu-1}{(H+1)^{2} \mu}\left[\frac{1-\alpha^{2}}{4(n-1) d^{2}}\left(\log \frac{\mu}{\mu-1}\right)^{2}-C\right] \leq \eta_{1} .
$$

It is clear that the left-hand side can be made to be positive by choosing $\mu$ close enough to 1 . The theorem is then proved by maximizing (4.5) with

$$
\frac{\mu}{\mu-1}=\exp \left[1+\left(1+\frac{4(n-1) d^{2}}{1-\alpha^{2}} C\right)^{\frac{1}{2}}\right] \text {. }
$$

This proves Theorem 1.1.

\section{ACKNOWLEDGMENTS}

The author would like to express his gratitude to Professor Peter $\mathrm{Li}$ and Professor Andrejs Treibergs for their many helpful suggestions and for their encouragement. He is especially indebted to Professor Peter Li, under whose advice this paper was completed.

\section{REFERENCES}

1. J. Cheeger, A lower bound for the smallest eigenvalue of the Laplacian, Problem in Analysis, Princeton Univ. Press, Princeton, NJ, 1970, pp. 195-199.

2. P. Li and S. T. Yau, Estimates of eigenvalues of a compact Riemannian manifold, Proc. Sympos. Pure Math., vol. 36, Amer. Math. Soc., Providence, RI, 1980, pp. 205-240.

3. L. E. Payne and I. Stakgold, On the mean value of the fundamental mode in the fuxed membrane problem. Applicable Anal. 3 (1973), 295-303.

4. L. E. Payne and H. Weinberger, The optimal Poincaré inequality for convex domains, Arch. Rational Mech. Anal. 5 (1960), 282-292.

5. F. W. Warner, Extension of the Rauch comparison theorem to submanifolds, Trans. Amer. Math. Soc. 122 (1966), 341-356.

Department of Mathematics, University of Toledo, Toledo, Ohio 43606

Current address: Department of Mathematics, University of Southern California, Los Angeles, California 90089 